\title{
Amplification of surface plasmon polariton wave in single-walled carbon nanotube using electric current pump
}

\author{
Sergey Moiseev ${ }^{1,2, *}$, Yuliya Dadoenkova $^{1,3}$, Aleksey Kadochkin ${ }^{1}$, and Igor Zolotovskii ${ }^{1}$ \\ ${ }^{1}$ Ulyanovsk State University, Ulyanovsk, 432017 Russia \\ ${ }^{2}$ Kotelnikov Institute of Radio Engineering and Electronics of the Russian Academy of Sciences, \\ Ulyanovsk Branch, Ulyanovsk, 432011 Russia \\ ${ }^{3}$ Donetsk Institute for Physics \& Technology, Donetsk, 83114, Ukraine
}

\begin{abstract}
We propose a surface plasmon polariton amplification technique based on direct energy transfer from a dc electric current flowing in a carbon nanotube. It is shown that when the synchronization conditions are satisfied, when the surface plasmon polariton wave velocity is close to the drift velocity of the charge carriers in the nanotube, the surface wave is significantly enhanced.
\end{abstract}

\section{Introduction and description of the system}

Surface waves propagating along the boundary between two media, one of which has a negative dielectric permittivity in the whole frequency range, are called surface plasmon polaritons (SPPs). The SPPs are characterized by high field localization and penetrate in each adjacent medium at a depth of the wavelength order, decaying exponentially with the distance from the interface. The investigation of SPPs is of great interest due to wide possibilities of their practical application. However, the use of materials with negative permittivity (as a rule, conductive media) inevitably leads to ohmic losses.

In this paper, we propose a new mechanism of the SPP amplification due to the transfer of electromagnetic energy from an external source (drift current wave) into a surface wave. In this case a necessary condition of the interaction of these waves is synchronization condition, when the phase velocity of the surface wave approaches the charge drift velocity. The main idea of this approach is described in details in our paper [1], devoted to amplification of a SPP wave in a semiconductor film with graphene layer. In this paper, the proposed approach is applied to a single-walled carbon nanotube (CNT).

The CNT is assumed to be an infinitely thin cylindrical shell of the length $L$ and radius $a=5 \mathrm{~nm}(L>>a)$ suspended in vacuum with voltage applied to its ends. We consider nonradiative TM-mode with non-zero component $E_{z}$ of electric field along the CNT. The presence of a radial component of the electric field is necessary to ensure the interaction of SPP wave with an electric pump current flowing along the CNT. The field distribution of SPP and spectral characteristics of CNT are derived within hydrodynamic model for electron

Corresponding author: serg-moiseev@yandex.ru 
gas in CNT and using Maxwell equations and general boundary conditions for electromagnetic field [2].

\section{Results of the numerical calculations}

The equation describing the interaction of the electromagnetic wave and the electron current $I_{d}$ writes:

$$
\frac{d E_{z}}{d z}+i \frac{\omega}{V_{p h}} E_{z}=-\frac{1}{2}\left(\frac{\omega}{V_{p h}}\right)^{2} 2 \frac{I_{d}\left|E_{0 z}\right|^{2}}{\beta^{2} P},
$$

where $V_{p h}=\omega / \beta, \omega$ and $\beta$ are phase velocity, angular frequency and propagation constant of SPP, and $P$ is power carried by SPP wave. Under influence of the SPP field the amplitude of the current is modulated along the CNT, and the current perturbation $\Delta I(x)=I(x)-I_{d}\left(I_{d}\right.$ is unperturbed current) can be described by the equation:

$$
\frac{d^{2} \Delta I}{d z^{2}}+2 i \frac{\omega}{V_{0}} \frac{d \Delta I}{d z}-\frac{1}{V_{0}^{2}}\left(\omega^{2}-\omega_{q}^{2}\right) \Delta I=i \frac{\omega}{V_{0}} \frac{I_{d}}{2 U_{0}} E_{z},
$$

where $U_{0}$ is potential difference, $V_{0}$ is charge carriers velocity in CNT, $\omega_{q}$ is reduced plasma frequency. Assuming that $\Delta I$ and $E_{z}$ change along the CNT proportionally to $\exp (-i G z), G$ is the harmonic perturbation wavenumber, on the basis of a joint solution of Eqs. (1) and (2), we calculate numerically the SPP amplification coefficient $\alpha=\operatorname{Im}(G)$.

The synchronism condition is fulfilled at frequency $\omega_{0}=3 \cdot 10^{13} \mathrm{rad} / \mathrm{s}$ (see the intersection of the solid blue and dashed green lines in Fig. 1(a)) corresponding to infrared regime. Within the frequency interval $\Delta \omega \approx 8 \cdot 10^{13} \mathrm{rad} / \mathrm{s}$ the SPP amplification coefficient $\alpha$ (Fig. 1(b)) is about $10^{6} \mathrm{~cm}^{-1}$ which is much larger than the SPP loss coefficient.
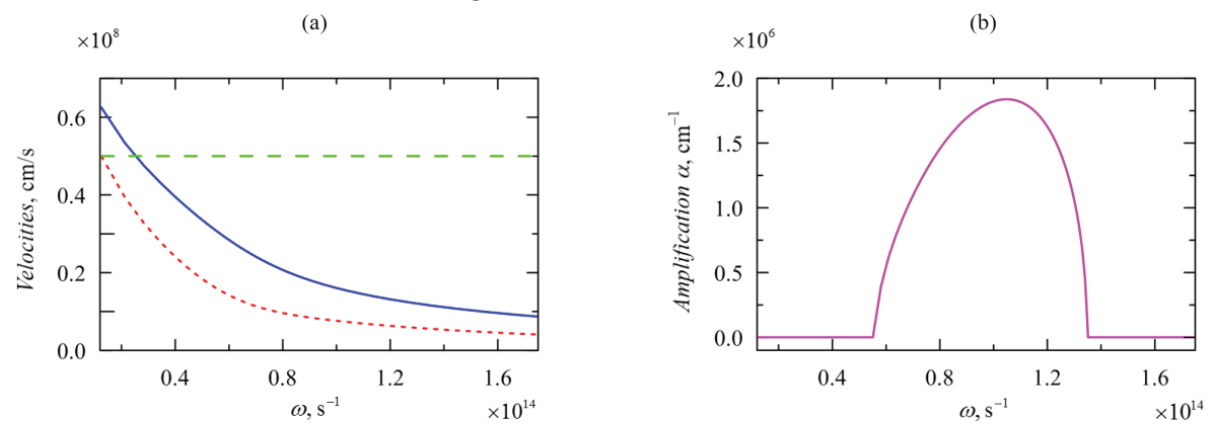

Fig. 1. Frequency dependences of: (a) SPP phase velocity (solid blue line) and group velocity (dotted red line), and drift current velocity $V_{0}=0.5 \cdot 10^{8} \mathrm{~cm} / \mathrm{s}$ (dashed green line) in CNT; (b) SPP amplification coefficient $\alpha$. The surface concentration of free electrons in CNT is $n_{s}=10^{12} \mathrm{~cm}^{-2}$.

This work was supported by the Ministry of Education and Science of the Russian Federation (Project No. 14.Z50.31.0015, State Contracts No. 3.7614.2017/P220, No. 3.5698.2017/P220, and No. 3.3889.2017), and the Russian Foundation for Basic Research (Project No. 17-02-01382).

\section{References}

[1] Y.S. Dadoenkova, S.G. Moiseev, A.S. Abramov, A.S. Kadochkin, A.A. Fotiadi, I.O. Zolotovskii, Ann. Phys. 529, 1700037 (2017)

[2] A. Moradi, Phys. Plasmas 21, 032106 (2014) 